\title{
Anti-allergic Effects of Caffeine in an Allergic Rhinitis Mouse Model
}

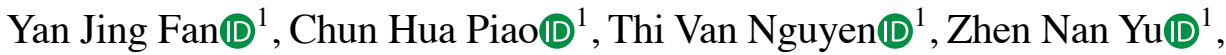 \\ Ok Hee Chai $\mathbb{D}^{1,2, *}$, Chang Ho Song $\mathbb{D}^{1,2, *}$ \\ ${ }^{1}$ Department of Anatomy, Jeonbuk National University Medical School, Jeonju, Jeonbuk 54896, Republic of Korea \\ ${ }^{2}$ Institute for Medical Sciences, Jeonbuk National University, Jeonju, Jeonbuk 54896, Republic of Korea
}

\begin{abstract}
Caffeine (1, 3, 7-trimethylxanthine) is one of the most widely consumed pharmacologically active products worldwide. Caffeine exhibits various pharmacological activities in central nervous, cardiovascular, and respiratory systems. Additionally, caffeine exhibited anti-inflammatory effects in lipopolysaccharide-challenged rats. However, to our knowledge, the potential anti-inflammatory activity of caffeine in allergic rhinitis (AR) has not yet been investigated. Therefore, in this study, we aimed to examine the anti-allergic effects of caffeine in ovalbumin (OVA)-induced AR in mice. We showed that caffeine attenuated the nasal symptoms, including rubbing and sneezing. It reduced the thickness of the nasal mucosa and alleviated goblet cell hyperplasia in the nasal mucosa. In addition, caffeine ameliorated the inflammation in the lungs and decreased OVA-specific immunoglobulin E (IgE) and IgG1 levels in the serum. It also reduced T helper type 2 (Th2) cytokine (interleukin (IL)-4 and IL-5) levels and elevated Th1 cytokine (IL-12 and IFN- $\gamma$ ) levels in the nasal lavage fluid. Collectively, we suggest that caffeine might have therapeutic effects in AR owing to its anti-inflammatory activities.
\end{abstract}

Keywords : Caffeine, Allergic rhinitis, Anti-allergic effects, Cytokines

\section{INTRODUCTION}

Allergic rhinitis (AR), a chronic upper airway disease, is characterized by nasal mucosa inflammation owing to the infiltration and activation of several inflammatory cells, such as mast cells, eosinophils, and lymphocytes [1]. Clinical symptoms, such as nasal itching, nasal congestion, sneezing, and clear nasal discharge, can significantly

\footnotetext{
*The authors equally contributed in this work.

The author(s) agree to abide by the good publication practice guideline for medical journals.

The author(s) declare that there are no conflicts of interest.

Received: January 7, 2020, Revised: January 9, 2020;

Accepted: January 14, 2020

Correspondence to: Ok Hee Chai (Department of Anatomy, Jeonbuk National University Medical School, 20 Gronji-ro, Jeonju 54907, Republic of Korea), Chang Ho Song (Department of Anatomy, Jeonbuk National University Medical School, 20 Gronji-ro, Jeonju 54907, Republic of Korea)

E-mail: okchai1004@jbnu.ac.kr, asch@jbnu.ac.kr
}

impede patients' quality of life and work performance [2]. Allergic diseases, including AR, are mediated by immunoglobulin $\mathrm{E}$ ( $\mathrm{IgE}$ ) and characterized by $\mathrm{T}$ helper type 2 (Th2)-mediated allergic inflammation. Thus, interleukin 4 (IL-4), IL-5, and IL-13 play important roles in the allergic responses [3]. IL-4 has been reported to induce Th2 cells class switching in B cells to immunoglobulin E ( $I g E)$ production, and the increased concentration of $\operatorname{IgE}$ has a pivotal role in allergic reactions [4,5]. IgG molecules have the unique feature of initiating pro- and anti-inflammatory reactions [6]. Th1 and Th2 are two of many distinct subsets of the $\mathrm{T}$ helper cells, defined by various functions and cytokine characterization [7]. An imbalance between Th1/ Th2 cells is suggested to contribute to the pathogenesis of allergic diseases, particularly AR [8]. Th2 cells were proposed to play important roles in the development of AR, and suppression of Th2 cells might be a new therapeutic 
<smiles>Cn1c(=O)c2c(ncn2C)n(C)c1=O</smiles>

Fig. 1. Structure of caffeine.

strategy for the treatment of AR [9]. Currently, effective approaches to alleviate AR symptoms include avoiding specific allergens, symptomatic drug treatment, and allergen immunotherapy (also known as allergy shots) to reduce patient sensitivity to allergens $[10,11]$. Symptomatic drug treatment includes antihistamines, decongestants, cromolyn sodium, leukotriene modifiers, nasal glucocorticoids, and nasal atropine [11]. However, avoiding known allergens does not significantly alleviate the symptoms. Antihistamines and corticosteroids have transient efficacy; moreover, their long-term use is associated with several side effects. Consequently, there is a need to develop safe and efficacious therapeutic alternatives for treatment of allergic disorders, including AR.

Caffeine (1, 3, 7-trimethylxanthine) is the most widely used psychostimulant in Western countries. It is found in coffee, tea, energy drinks, several soft drinks, and cocoa [12]. The structure of caffeine is shown in Fig. 1. Caffeine exhibits various pharmacological activities in central nervous, cardiovascular, and respiratory systems. It acts as an adenosine receptor antagonist, phosphodiesterase inhibitor, and ryanodine receptor activator [13]. Goto et al. demonstrated that in an allergic mice model, coffee could induce a Th1-type response of immune system and prevent allergy developing [14]. Caffeine has been shown to exert several biological effects, including antioxidant, antiaging, and anti-obesity effects $[15,16]$. Additionally, caffeine exhibited anti-inflammatory effects in lipopolysaccharide-challenged rats [17]. However, to our knowledge, the potential anti-inflammatory activity of caffeine in AR has not yet been investigated. Thus, in this study, we aimed to evaluate the anti-allergic effects of caffeine in an ovalbumin (OVA)-induced AR mouse model. More importantly, the present study found that caffeine could attenuated nasal symptoms of the AR model, downregulation the expression of Th1 cytokines, increased Th2-related cytokines and alleviated the inflammation in nasal mucosa and lung. Thus, we hypothesized that caffeine may be an effective reagent for the therapeutic of AR.

\section{MATERIALS AND METHODS}

\section{Animals}

Six-week old male BALB/c mice were purchased from Damool Science (Daejeon, Korea). The animals were housed in an air-conditioned room under a 12-h light/dark cycle. All animal experiments were performed in accordance with the guidelines for Animals Care and Use of Jeonbuk National University Laboratory Animal Center (CBN 2019-071).

\section{AR murine model establishment and caffeine treatment}

The mice were divided into six groups $(n=6)$ as follows: control group, OVA group, caffeine (Sigma-Aldrich, St. Louis, MO, USA) 5,10 , and $20 \mathrm{mg} / \mathrm{kg}$ treatment groups, and dexamethasone (Dex; Sigma-Aldrich, St. Louis, MO, USA) $2.5 \mathrm{mg} / \mathrm{kg}$ treatment group. The mice were intraperitoneally injected with $50 \mu \mathrm{g}$ of OVA (grade VI; Sigma-Aldrich, St. Louis, MO, USA) and $1 \mathrm{mg}$ of alum (Thermo Scientific, Rockford, IL, USA) on days 1, 8, and 15 to induce systemic sensitization. On days 22 to 28 , mice in the OVA, caffeine, and Dex groups were challenged with $20 \mu \mathrm{L}$ of 10 $\mathrm{mg} / \mathrm{mL}$ OVA solution instilled into each nasal cavity [18]. From days 16 to 28 , mice in the caffeine and Dex groups received $20 \mu \mathrm{L}$ of caffeine $(5,10$, or $20 \mathrm{mg} / \mathrm{kg}$ ) or Dex $(2.5$ $\mathrm{mg} / \mathrm{kg}$ ) by intranasal instillation (Fig. 2) [18]. Mice in the OVA group received the same volume of saline. Mice in the control group were treated with saline without OVA sensitization and challenge. Twenty-four hours after the last OVA challenge, the mice were sacrificed and samples were collected for further assays.

\section{Monitoring of the nasal symptoms}

The mice were intranasally challenged with $10 \mathrm{mg} / \mathrm{mL}$ OVA ( $20 \mu \mathrm{L} /$ nasal cavity) for 7 consecutive days from day 22. After the last challenge, nasal rubbing (or itching) and sneezing behaviors were recorded for $15 \mathrm{~min}$ and then counted by blinded experimenters. 


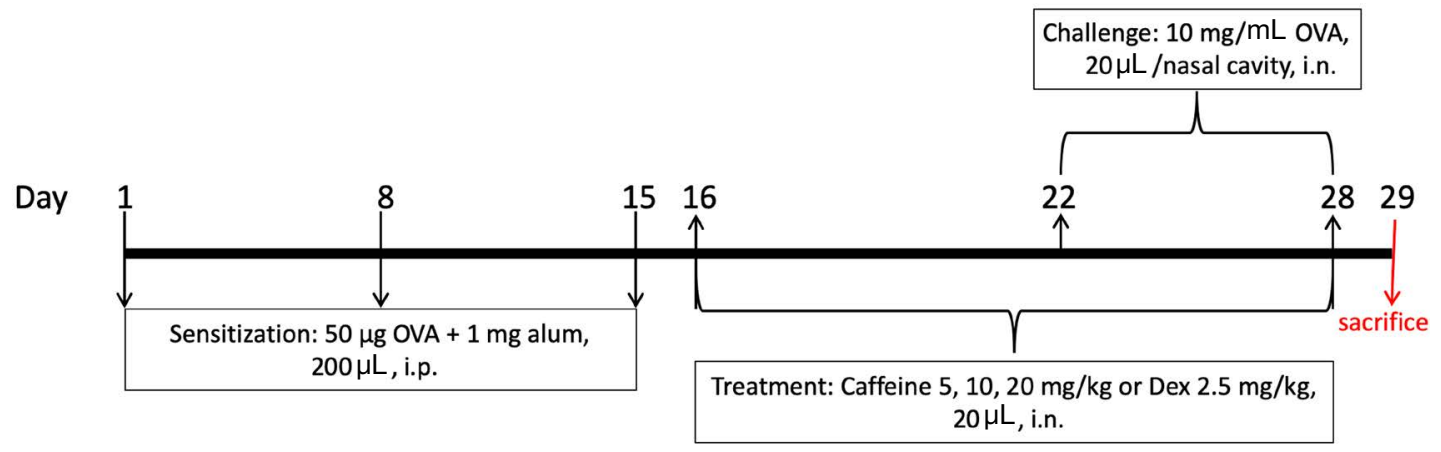

Fig. 2. Experimental timeline of allergic rhinitis induction and caffeine treatment. Mice in the OVA group, caffeine groups, and dexamethasone (Dex) group were sensitized with ovalbumin (OVA) on days 1,8 , and 15 and then challenged with OVA on days 22 to 28 . Mice in the caffeine groups and Dex group received $20 \mu \mathrm{L}$ of caffeine $(5,10$, or $20 \mathrm{mg} / \mathrm{kg})$ or Dex $(2.5 \mathrm{mg} / \mathrm{kg})$ by intranasal instillation for 13 consecutive days from days 16 to 28 . Mice in the OVA group received the same volume of saline instead of caffeine or Dex. Mice in the control group were treated with saline without OVA sensitization and challenge. Mice were sacrificed $24 \mathrm{~h}$ after the last OVA challenge on day 29.

\section{Collection of serum and nasal lavage fluid (NALF) and cell counting in NALF}

On day 29, $24 \mathrm{~h}$ after the last OVA challenge, the mice were anesthetized using diethyl ether and sacrificed. Blood samples were collected via orbital puncture and centrifuged to obtain serum. NALF was collected using an 18-gauge catheter. The trachea was partially resected, and a catheter was inserted from the trachea into the nasopharynx. The nasal passages were gently perfused with $1 \mathrm{~mL}$ of saline. NALF was centrifuged (Gyro 1730 MR, GYROZEN, Korea) at $1000 \mathrm{~g}$ for $10 \mathrm{~min}$ at $4^{\circ} \mathrm{C}$, and the supernatant was collected for cytokine assay. The supernatants were stored at $-80^{\circ} \mathrm{C}$ until use in the enzyme-linked immunosorbent assay (ELISA).

To determine differential cell counts, $150 \mu \mathrm{L}$ of NALF was centrifuged onto the sliders using a cytospin device (Centrifuge 5403, Eppendorf, Hamburg, Germany) at 100 $\mathrm{g}$ for $10 \mathrm{~min}$. Then, the sliders were stained with the DiffQuik staining reagent (1-5-1 Wakinohama-Kaicaffeinendori, Chou-Ku, Kobe, Japan) for cell staining, according to the manufacturer's instructions. The numbers of cells, including eosinophils, macrophages, neutrophils, and lymphocytes, were counted using a light microscope.

\section{Measurement of OVA-specific immunoglobulin E (IgE), IgG1, and IgG2a levels in the serum}

After sacrifice, blood samples were collected and centrifuged at $1000 \mathrm{~g}$ for $10 \mathrm{~min}$ at $4^{\circ} \mathrm{C}$ to obtain serum. The levels of OVA-specific IgE, IgG1, and IgG2a in the serum were measured using ELISA kits (BioLegend, San Diego,
CA, USA, Catalog No. 439807; Cayman, Ann Arbor, MI, USA, Catalog No. 500830; and Chondrex, Redmond, WA, USA, Catalog NO. 3015, respectively), according to the manufacturers' instructions.

\section{Measurement Th1 and Th2 cytokine level in the NALF}

The levels of Th1 cytokines, including interferon-gamma (IFN- $\gamma$; R\&D Systems, St. Paul, MI, USA, Catalog No. MIF00) and IL-12 (R\&D Systems, St. Paul, MI, USA, Catalog No. M1270), and Th2 cytokines, including IL-4 (R\&D Systems, St. Paul, MI, USA, Catalog No. M40000B) and IL-5 (R\&D Systems, St. Paul, MI, USA, Catalog No. $\mathrm{M} 5000$ ), in the NALF were quantified using cytokine assay kits, according to the manufacturer's instructions.

\section{Histopathological examination of the lungs}

After sacrifice, the lungs were removed and fixed in $10 \%$ formalin. Histopathological staining of the lungs was performed according to routine procedures. The specimen was dehydrated using ethyl alcohol and xylene. Tissues were embedded in paraffin and sliced into $5-\mu \mathrm{m}$ thick sections. The sections were stained with hematoxylin and eosin (H\&E) to observe inflammatory cell infiltration and the microscopic architecture of the lungs. Mucus secretion was quantified by periodic acid-Schiff(PAS) staining [18].

\section{Histopathological examination of the nasal tissue}

Nasal tissue for histopathological examination was fixed in $10 \%$ formalin for 3 days at room temperature. Then, the 
heads were decalcified in an ethylenediaminetetraacetic acid decalcifying solution for 6 days at room temperature [18]. The specimen was dehydrated using ethyl alcohol and xylene and then embedded in paraffin. The samples were sliced into $4-\mu \mathrm{m}$ thick sections and stained with H\&E to evaluate overall inflammation. Some sections were stained with PAS to observe goblet cell hyperplasia.

\section{Statistical analysis}

Statistical analyses were performed using GraphPad Prism software (v 5.0; La Jolla, CA, USA). Data are expressed as the means \pm standard error of the mean (SEM). The differences among groups were analyzed by one-way analysis of variance (ANOVA), followed by Tukey's posthoc test. Differences were considered statistically significant at $P<0.05$.

\section{RESULTS}

\section{Caffeine ameliorated nasal symptoms}

To investigate the anti-allergic effects of caffeine, OVA was used to induce AR in mice; then, the frequency of nose rubbing and sneezing was counted for 15 min after the last OVA intranasal challenge. Mice in the OVA-induced AR group showed a significant increase in the number of instances of nose rubbing and sneezing after intranasal OVA challenge, compared with that in the control group. Caffeine administration significantly alleviated allergic nasal symptoms in a dose-dependent manner, compared with

(A)

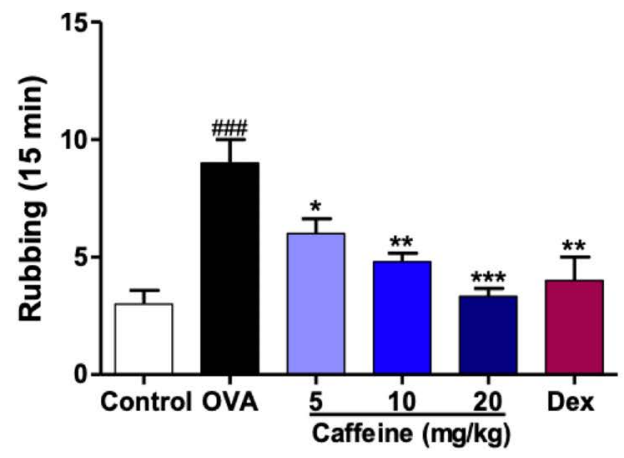

those in the OVA group. Similarly, Dex treatment significantly inhibited allergic symptoms in the AR mouse model (Fig. 3A and B).

\section{Caffeine reduced inflammatory cell infiltration in the NALF}

To investigate the effects of caffeine on nasal inflammation in the AR mouse model, the number of total and differential inflammatory cells in the NALF was counted. The number of total inflammatory cells, including eosinophils, neutrophils, and macrophages, significantly increased in the OVA-induced AR mice, compared with that in the control mice. However, caffeine at 5, 10, and $20 \mathrm{mg} / \mathrm{kg}$ and Dex at $2.5 \mathrm{mg} / \mathrm{kg}$ significantly decreased the number of inflammatory cells in the NALF, compared with that in the OVA group (Fig. 4A and B). In addition, NALF cytospin preparations showed an increase in epithelial cells (black arrows) and eosinophils (red arrows) in the OVA group, which were reduced in the caffeine- and Dex-treated mice (Fig. 4C).

\section{Caffeine alleviated OVA-induced histopathological changes in the lung tissue}

To evaluate the anti-inflammatory effects of caffeine in OVA-induced AR mice, lung tissue sections were stained with $H \& E$ and PAS stains. Histopathological changes, such as marked mucus production in the bronchial lumen, goblet cell hyperplasia in the bronchial epithelium, and inflammatory cell infiltration in the perivascular and bronchial areas, were observed in lung tissue sections from the OVA-induced AR mice (Fig. 5A and B). However, both caffeine and Dex

\section{(B)}

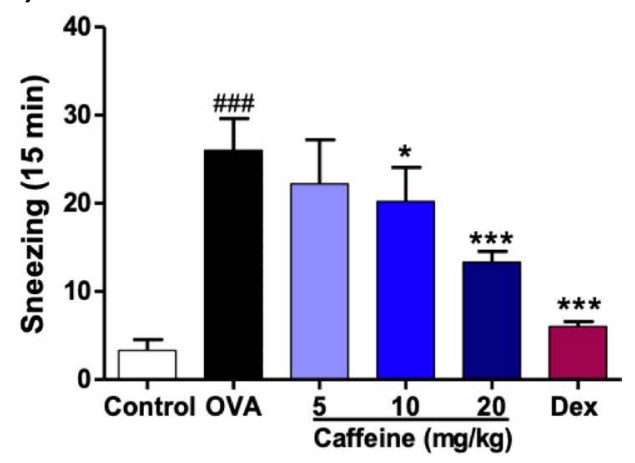

Fig. 3. Monitoring of the nasal symptoms in the allergic rhinitis murine model. (A) Nasal rubbing and (B) sneezing scores. The values represent the means $\pm \operatorname{SEM}\left(n=6 /\right.$ group). Significant differences at ${ }^{\# \# \#} P<0.001$ compared with the control group, ${ }^{* * *} P<0.001, * * P<0.01$, and $* P<0.05$ compared with the ovalbumin (OVA) group. Dex: dexamethasone. 
(A)

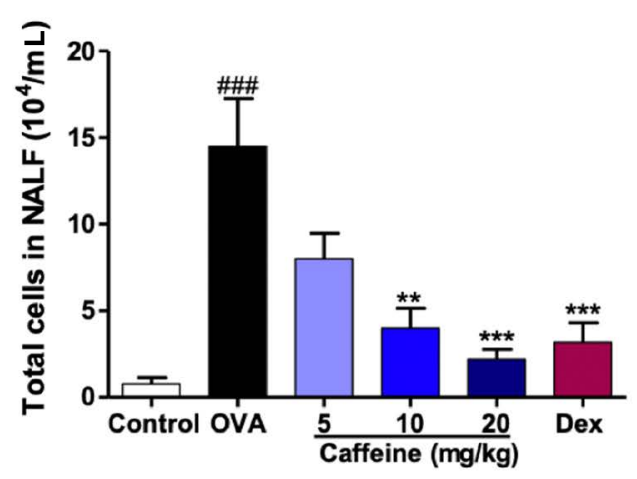

(C)
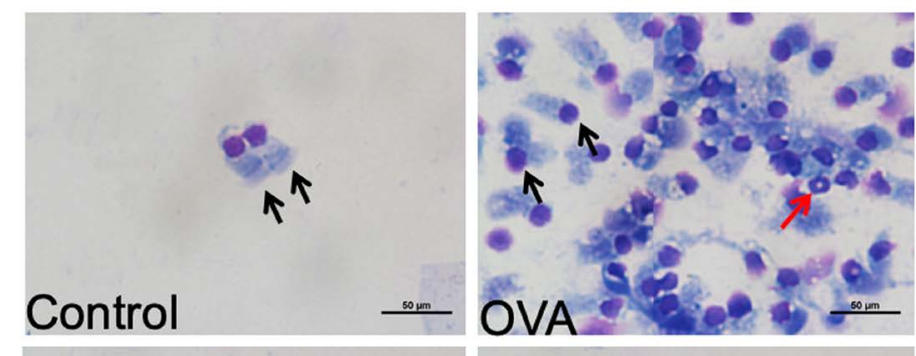

(B)

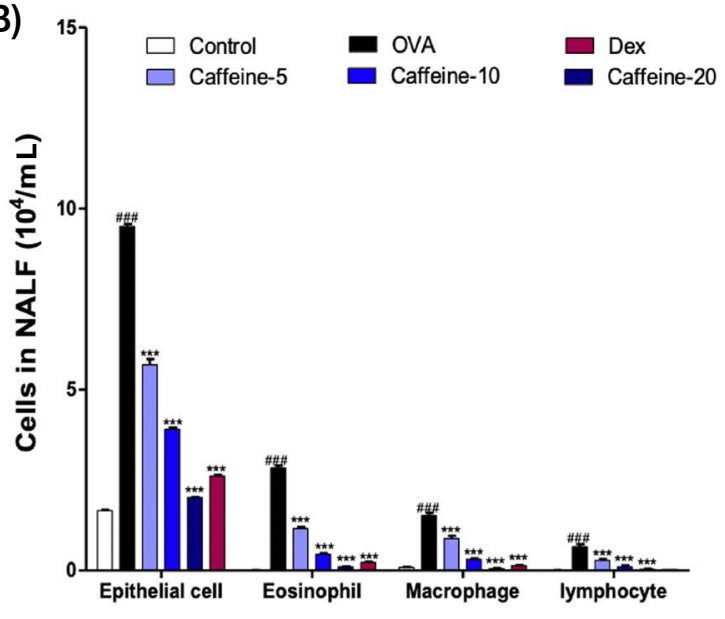

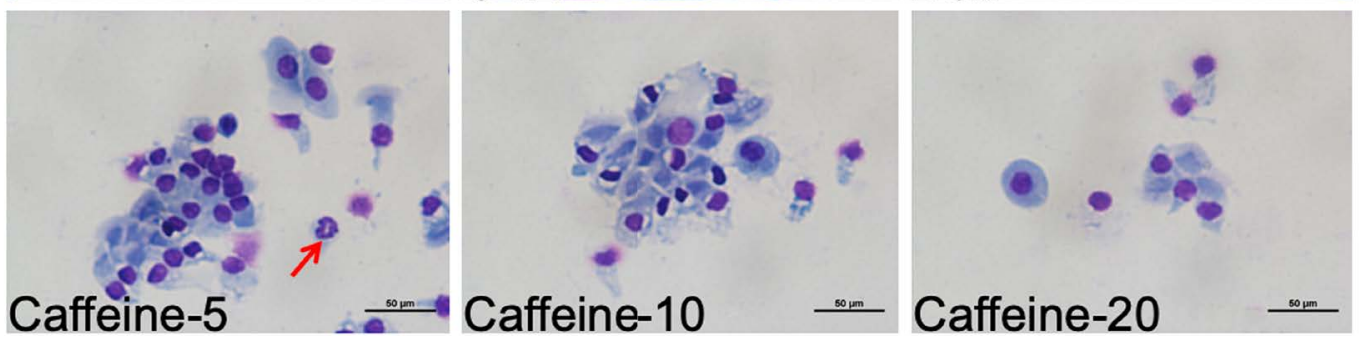

Fig. 4. The number of total cells (A), differential cells (B), and a photograph of cytospin cells (C) in the nasal lavage fluid (NALF). Cytospin cell preparations were obtained by adding NALF onto clean glass slides; then, the cells were stained with Diff-Quik stain. Red and black arrows indicate eosinophils and epithelial cells respectively. The values represent the means \pm SEM ( $n=6 /$ group). Significant differences at $* P<0.05$, $* * P<0.01$, and $* * * P<0.001$ compared with the ovalbumin (OVA) group. Bars $=50 \mu$ m. Dex: dexamethasone.

significantly alleviated these histopathological changes.

\section{Caffeine decreased nasal mucosa thickness and goblet cell hyperplasia}

After sacrifice, samples of the mouse heads were collected. The sectioned slides were stained with H\&E to examine the overall structure. The thickness of the nasal mucosa obviously increased in the OVA group, compared with that in the control group, whereas it significantly decreased in the mice treated with caffeine or Dex (Fig. 6A). Goblet cell hyperplasia was evaluated based on PAS staining. The hyperplasia goblet cells, indicated by red arrows, were more pronounced than in the control group; however, both caffeine and Dex treatment resulted in reducing this phenomenon (Fig. 6B).

\section{Caffeine reduced OVA-specific antibody levels in the serum}

After sacrifice, blood samples were collected and centrifuged to obtain the serum. The levels of OVA-specific IgE, IgG1, and IgG2a were measured using ELISA kits. The OVA-specific IgE level was higher in the OVA-challenged AR mice than in the control mice. Treatment with caffeine and Dex reduced the OVA-specific IgE level in the serum 

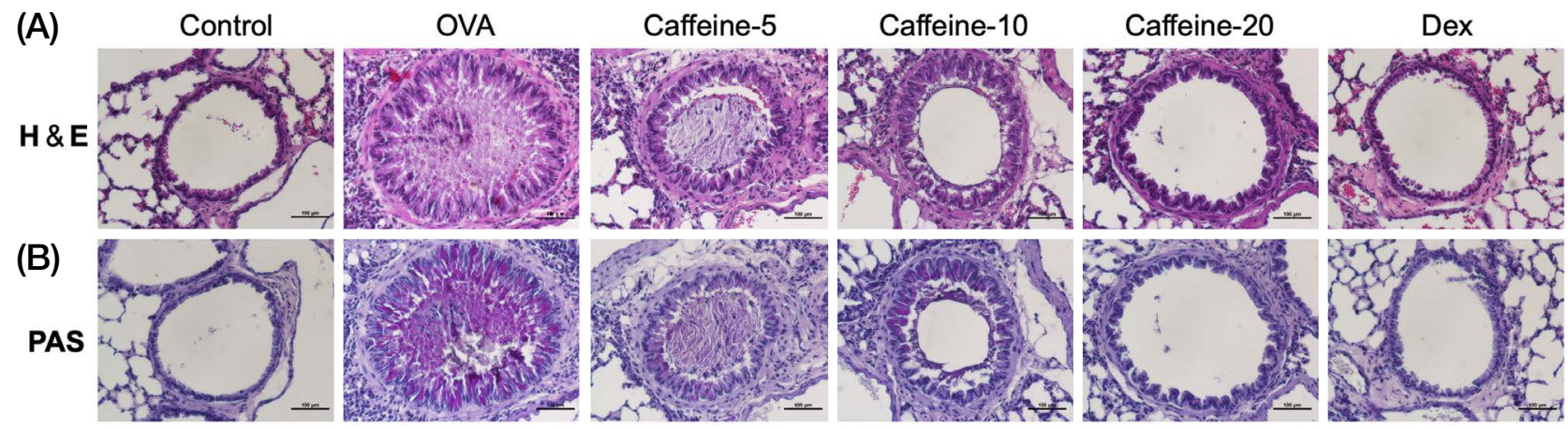

Fig. 5. Histopathological changes in the lung tissues. (A) Hematoxylin and eosin (H\&E) staining. (B) Periodic acid-Schiff (PAS) staining. The sections showed inflammation in the lung tissue of OVA-challenged mice, which was significantly alleviated by caffeine treatment. Bars $=100 \mu \mathrm{m}$. Dex: dexamethasone, OVA: ovalbumin.

(A)

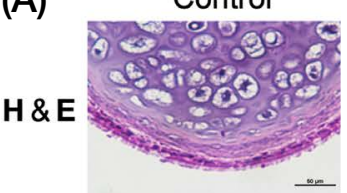

(B)

PAS

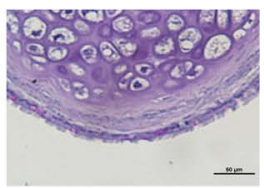

OVA
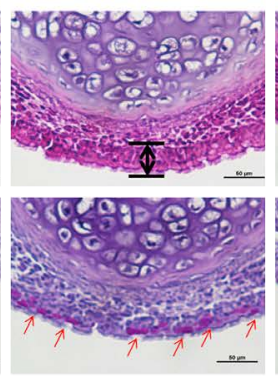

Caffeine-5
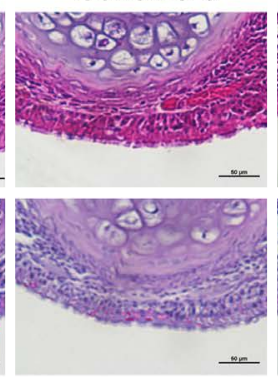

Caffeine-10
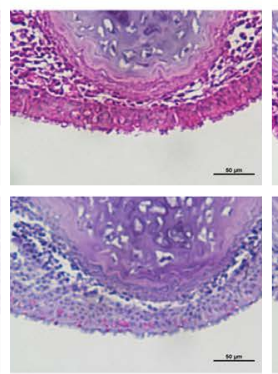

Caffeine-20
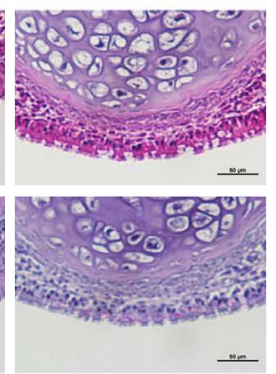

Dex

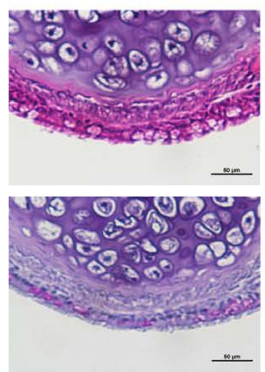

Fig. 6. Histopathological changes in the nasal tissues. (A) The thickness of the nasal mucosa, H\&E staining. (B) Goblet cell hyperplasia, PAS staining. Black both arrow represents epithelial thickness. Red arrows indicate goblet cells. Bars $=50 \mu \mathrm{m}$.
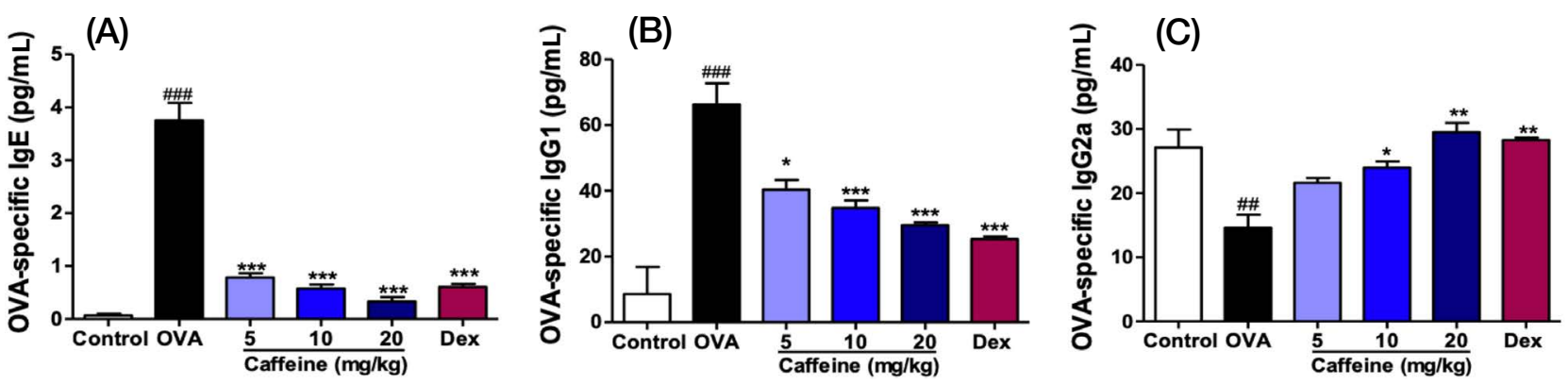

Fig. 7. The levels of ovalbumin (OVA)-specific antibodies in the serum. (A) OVA-specific IgE level. (B) OVA-specific IgG1 level. (C) OVA-specific IgG2a level. The levels of OVA-specific IgE, IgG1, and IgG2a in the serum were measured using ELISA kits. The values represent the means $\pm \operatorname{SEM}(n=6 /$ group). Significant differences at $* P<0.05, * * P<0.01$, and $* * * P<0.001$ compared with the ovalbumin $(\mathrm{OVA})$ group. Dex: dexamethasone.

(Fig. 7A). Similarly, the OVA-specific IgG1 level increased in the OVA group, compared with that in the control group, but decreased in the caffeine- and Dex-treated groups (Fig. 7B). In addition, the OVA-specific IgG2a level significantly decreased in the OVA group; however, it recovered after treatment with caffeine or Dex (Fig. 7C).

\section{Caffeine modulated Th1 and Th2 cytokine levels in the NALF}

The NALF was collected after the mice were sacrificed, and the levels of Th1 cytokines (IFN- $\gamma$ and IL-12) were measured using ELISA kits. IFN- $\gamma$ and IL-12 levels signifi- 
(A)

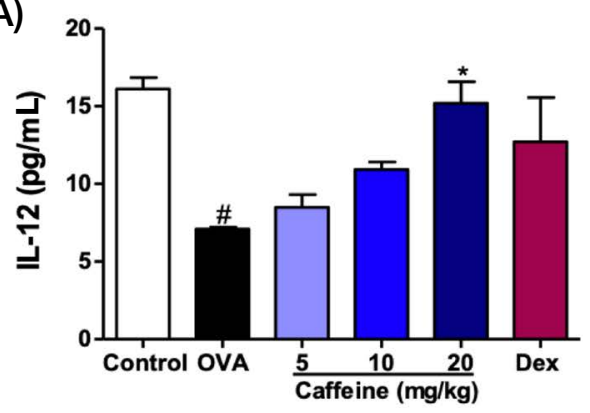

(C)

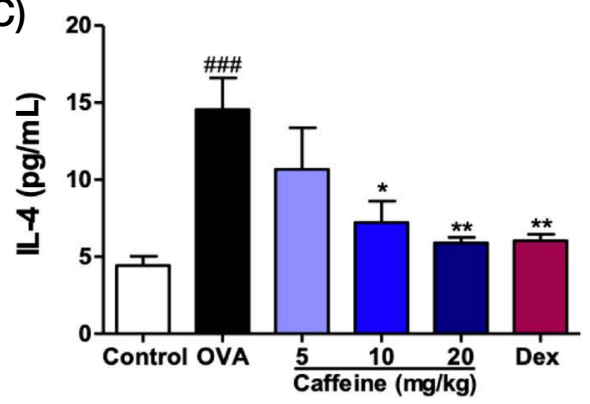

(B)

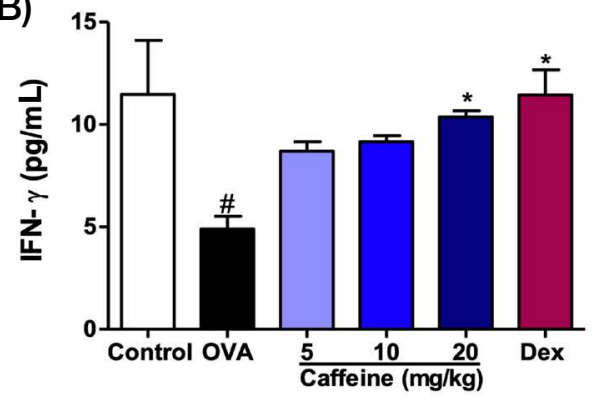

(D)

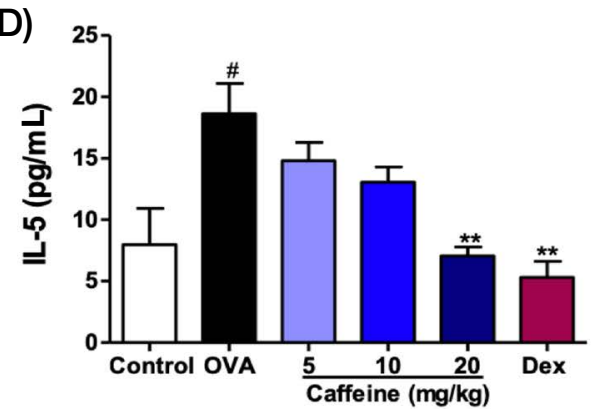

Fig. 8. The levels of Th1 and Th2 cytokines in the NALF. (A) IFN- $\gamma$ level. (B) IL-12 level. (C) IL-4 level. (D) IL-5 level. The levels of cytokines in the NALF were measured using ELISA kits. The values represent the means \pm SEM ( $n=6 /$ group). Significant differences at $* P<0.05, * * P<0.01$, and $* * * P<0.001$ compared with the ovalbumin (OVA) group. Dex: dexamethasone.

cantly decreased in the OVA-induced AR mice, compared with those in the control group; however, caffeine or Dex treatment significantly increased the levels of Th1 cytokines (Fig. 8A and B).

To investigate the effects of caffeine on Th2 cytokine levels in the NALF, IL-4 and IL-5 levels were measured using ELISA kits. OVA-induced mice exhibited an increase in Th2-related cytokine levels, compared with those in the control group. However, treatment with caffeine or Dex significantly reduced the levels of IL-4 and IL-5 (Fig. 8C and D).

\section{DISCUSSION}

Caffeine, the major pharmacologically active constituent in coffee, is a non-selective adenosine receptor antagonist. It showed systemic anti-inflammatory effects in previous studies $[19,20]$. Alfaro et al. showed that coffee consumption had some positive effects on the respiratory system [21]. However, the potential anti-inflammatory effects of caffeine have not yet been investigated in an experimental model of AR.

In this study, we showed that caffeine significantly allevi- ated AR symptoms, reduced AR biomarkers, and decreased inflammatory cytokine levels. Caffeine markedly decreased the number of instances of nose rubbing and sneezing, compared with that in the AR group. Additionally, it reduced the total and differential inflammatory cells, including eosinophils, macrophages, and lymphocytes, in the NALF. Moreover, caffeine treatment reduced inflammatory cell infiltration and goblet cell hyperplasia in the nasal tissues. It also decreased the levels of inflammatory cytokines in the NALF and serum of OVA-induced AR mice.

AR is characterized by an increase in the numbers of Th2 cells and Th2-related cytokines and a decrease in the number of Th1 cells and Th1-related cytokines [22]. Th2-related cytokines, including IL-4 and IL-5, are responsible for IgE production, whereas Th1-related cytokines are associated with the suppression of IgE synthesis $[22,23]$. Therefore, the reduction in the OVA-specific IgE level in the caffeine-treated groups might be attributed to the decrease in Th2 cytokines and the increase in Th1 cytokines. In allergic diseases such as AR, Th2 cells induce allergen-specific IgE release from the $\mathrm{B}$ cells and promote eosinophil and mast cell infiltration into the target tissues [24]. Activated Th2 cells produce IL- 4 and IL-5, which are involved in the symptoms of allergic diseases [24]. IL-4 increases IgE syn- 
thesis in the B cells and participates in the allergic reaction [25]. IL-5 is a major differentiation and maturation factor of eosinophils; simultaneously, it is crucial for eosinophil activation, development, survival, and response to other cytokines [26]. Our results showed that caffeine reduced the levels of Th2 cytokines, such as IL-4 and IL-5, resulting in relief of AR clinical symptoms. Therefore, we suggested that caffeine exerted anti-allergic effects via regulation of $\mathrm{Th} 2$ cytokines in AR responses.

IFN- $\gamma$ is an important Th1 effector cytokine, which affects Th1/Th2 differentiation, triggers the production of macrophages, and inhibits Th2 cell proliferation [27]. Our resulted showed that the levels of Th1-related cytokines, such as IFN- $\gamma$ and IL-12, increased in the caffeine group, compared with those in the OVA group, providing evidence that caffeine could shift Th2 to Th1 immune response.

As Igs may play important roles in mediating allergy and inflammatory reactions, we measured the levels of several Ig antibodies (IgE, IgG1, and IgG2a) that have been implicated in the B-cell immune responses modulated by cytokines from Th cells [28]. In this study, OVA-specific IgG2a levels increased in the caffeine and Dex-treated groups, suggesting that caffeine might activate the Th1 immune response. The production of OVA-specific $\operatorname{IgE}$ and $\mathrm{IgG1}$ significantly increased in the OVA group, compared with that in the control group. However, treatment with caffeine significantly reduce both OVA-specific IgE and IgG1 production, suggesting that caffeine might attenuate Th2 immune responses.

In AR, infiltration of various inflammatory cells occurs, inducing airway and nasal inflammation. In this study, we showed that OVA induced histopathological changes in the lung and nasal tissues, increased nasal mucosa thickness, and resulted in goblet cell hyperplasia in the nasal mucosa. However, caffeine treatment could reverse all these histopathological changes.

There are many mechanisms involved in the development of AR and understanding these mechanisms can be helpful in the treatment of rhinitis. Exploring the mechanisms that caffeine may be involved in could shed more light on how caffeine relievers AR. More works are needed to do in this area.

Collectively, this study showed that intranasally applied caffeine significantly attenuated inflammation in the OVA-induced AR model. Caffeine alleviated AR symptoms, reduced the thickness of the nasal mucosa, attenuated goblet cell hyperplasia in the nasal mucosa, decreased IL-4 and IL-5 levels in the NALF, reduced OVA-specific IgE and IgG1 levels in the serum, increased IFN- $\gamma$ and IL-12 levels in the NALF, and elevated OVA-specific IgG2a levels in the serum. Collectively, these results suggested that caffeine might be used as a therapeutic agent in AR.

\section{REFERENCES}

1. Wu S, Tang S, Peng H, Jiang Y, Liu Y, Wu Z, et al. Effects of lentivirus-mediated CCR3 RNA interference on the function of mast cells of allergic rhinitis in mice. Int Immunopharmacol. 2019 Nov 24. doi: 10.1016/j.intimp.2019.106011.

2. Xiao B, Wang JH, Zhou CY, Chen JM, Zhang N, Zhao N, et al. Ethno-medicinal study of Artemisia ordosica Krasch. (traditional Chinese/Mongolian medicine) extracts for the treatment of allergic rhinitis and nasosinusitis. J Ethnopharmacol. 2020 Feb 10. doi: 10.1016/j.jep.2019.112262.

3. Zhang YL, Shin HJ, Lee JH, Lee J. Antiallergic effect of Hizikia fusiformis in an ovalbumin-induced allergic rhinitis mouse model. Clin Exp Otorhinolaryngol. 2019;12:196-205.

4. Xu W, Hu M, Zhang Q, Yu J, Su W. Effects of anthraquinones from Cassia occidentalis L. on ovalbumin-induced airways inflammation in a mouse model of allergic asthma. J Ethnopharmacol. 2018;221:1-9. doi: 10.1016/j.jep.2018.04.012.

5. Lee JW, Min JH, Kim MG, Kim SM, Kwon OK, Oh TK et al. Pistacia weinmannifolia root exerts a protective role in ovalbumininduced lung inflammation in a mouse allergic asthma model. Int J Mol Med. 2019;44:2171-80. doi: 10.3892/ ijmm.2019.4367.

6. Aschermann S, Lux A, Baerenwaldt A, Biburger M, Nimmerjahn $F$. The other side of immunoglobulin G: suppressor of inflammation. Clin Exp Immunol. 2010;160:161-7. doi: 10.1111/j.1365-2249.2009.04081.x.

7. Mosmann TR, Coffman RL. TH1 and TH2 cells: different patterns of lymphokine secretion lead to different functional properties. Annu Rev Immunol. 1989;7:145-73.

8. Kirmaz C, Bayrak P, Yilmaz O, Yuksel H. Effects of glucan treatment on the Th1/Th2 balance in patients with allergic rhinitis: a double-blind placebo-controlled study. Eur Cytokine Netw. 2005;16:128-34.

9. Shirasaki H, Kanaizumi E, Seki N, Himi T. Correlation of local FOXP3-expressing $\mathrm{T}$ cells and Th1-Th2 balance in perennial allergic nasal mucosa. Int J Otolaryngol. 2011. doi: $10.1155 / 2011 / 259867$.

10. Brozek JL, Bousquet J, Agache I, Agarwal A, Bachert C, Bosnic-Anticevich S, et al. Allergic Rhinitis and its Impact on Asthma (ARIA) guidelines-2016 revision. J Allergy Clin 
Immunol. 2017;140:950-8.

11. Quraishi SA, Davies MJ, Craig TJ. Inflammatory responses in allergic rhinitis: traditional approaches and novel treatment strategies. J Am Osteopath Assoc. 2004;104:S7-15.

12. Kolahdouzan M, Hamadeh MJ. The neuroprotective effects of caffeine in neurodegenerative diseases. CNS Neurosci Ther. 2017;23:272-90.

13. Tatler AL, Barnes J, Habgood A, Goodwin A, McAnulty RJ, Jenkins G. Caffeine inhibits TGFbeta activation in epithelial cells, interrupts fibroblast responses to TGFbeta, and reduces established fibrosis in ex vivo precision-cut lung slices. Thorax. 2016;71:565-7.

14. Goto M, Yamaki K, Shinmoto H, Takano-Ishikawa Y. Continuous orally administered coffee enhanced the antigen-specific Th1 response and reduced allergic development in a TCR-transgenic mice model. Biosci Biotechnol Biochem. 2009;11:2439-44

15. Tiwari KK, Chu C, Couroucli X, Moorthy B, Lingappan $K$. Differential concentration-specific effects of caffeine on cell viability, oxidative stress, and cell cycle in pulmonary oxygen toxicity in vitro. Biochem Biophys Res Commun. 2014;450:1345-50.

16. Hosoi T, Toyoda K, Nakatsu K, Ozawa K. Caffeine attenuated ER stress-induced leptin resistance in neurons. Neurosci Lett. 2014;569:23-6.

17. Hwang JH, Kim KJ, Ryu SJ, Lee BY. Caffeine prevents LPS-induced inflammatory responses in RAW264.7 cells and zebrafish. Chem Biol Interact. 2016;248:1-7.

18. Bui TT, Kwon DA, Choi DW, Jung SY, Lee SY, Piao CH, et al. Rosae multiflorae fructus extract and its four active components alleviate ovalbumin-induced allergic inflammatory responses via regulation of $\mathrm{Th} 1 / \mathrm{Th} 2$ imbalance in $\mathrm{BALB} / \mathrm{c}$ rhinitis mice. Phytomedicine. 2019;55:238-48.
19. Fredholm BB, AP IJ, Jacobson KA, Klotz KN, Linden J. International Union of Pharmacology. XXV. Nomenclature and classification of adenosine receptors. Pharmacol Rev. 2001;53:527-52.

20. Köroğlu OA, MacFarlane PM, Balan KV, Zenebe WJ, Jafri A, Martin RJ, et al. Anti-inflammatory effect of caffeine is associated with improved lung function after lipopolysaccharide-induced amnionitis. Neonatology. 2014;106:235-40.

21. Alfaro TM, Monteiro RA, Cunha RA, Cordeiro CR. Chronic coffee consumption and respiratory disease: A systematic review. Clin Respir J. 2018;12:1283-94.

22. Chung F. Anti-inflammatory cytokines in asthma and allergy: interleukin-10, interleukin-12, interferon-gamma. Mediators Inflamm. 2001;10:51-9.

23. Cameron L, Hamid Q, Wright E, Nakamura Y, Christodoulopoulos P, Muro S, et al. Local synthesis of epsilon germline gene transcripts, IL-4, and IL-13 in allergic nasal mucosa after ex vivo allergen exposure. J Allergy Clin Immunol. 2000;106:46-52.

24. Romagnani S. Cytokines and chemoattractants in allergic inflammation. Mol Immunol. 2002;38:881-5.

25. Deo SS, Mistry KJ, Kakade AM, Niphadkar PV. Role played by Th2 type cytokines in IgE mediated allergy and asthma. Lung India. 2010;27:66-71.

26. Coffman RL, Seymour BW, Hudak S, Jackson J, Rennick D. Antibody to interleukin-5 inhibits helminth-induced eosinophilia in mice. Science. 1989;245:308-10.

27. Kidd P. Th1/Th2 balance: the hypothesis, its limitations, and implications for health and disease. Altern Med Rev. 2003;8:223-46.

28. Zhao N, Liu Y, Liang H, Jiang X. Bone marrow-derived mesenchymal stem cells reduce immune reaction in a mouse model of allergic rhinitis. Am J Transl Res. 2016;8:5628-36. 